\title{
Towards a framework for investigating tangible environments for learning
}

\author{
Sara Price \\ RCUK Academic Fellow \\ London Knowledge Lab, Institute of Education \\ 23-29 Emerald Street \\ London, UK. WC1N 3QS \\ E-mail: s.price@ioe.ac.uk \\ Fax number: 02077632138 \\ Contact Author \\ Jennifer G. Sheridan \\ Research Officer \\ London Knowledge Lab, Institute of Education \\ 23-29 Emerald Street \\ London, UK. WC1N 3QS \\ E-mail: j.sheridan@ioe.ac.uk \\ Fax number: 02077632138 \\ Taciana Pontual Falcão \\ Research Officer \\ London Knowledge Lab, Institute of Education \\ 23-29 Emerald Street \\ London, UK. WC1N 3QS \\ E-mail: t.pontual@ioe.ac.uk \\ Fax number: 02077632138

\section{George Roussos} \\ Senior Lecturer \\ London Knowledge Lab, Department of Computer Science, Birkbeck College \\ 23-29 Emerald Street \\ London, UK. WC1N 3QS \\ E-mail: g.roussos@dcs.bbk.ac.uk \\ Fax number: 02077632138
}

\section{Biographical notes}

\section{Sara Price}

Sara is a Senior Researcher and RCUK Academic Fellow based at the London Knowledge Lab, Institute of Education. Sara holds a BA and a DPhil in Psychology, both from the University of Sussex. Before joining the London Knowledge Lab, Sara was a research fellow on the EPSRC funded EQUATOR project. Her research focuses on the role of representation and technology in mediating learning, particularly within the area of External Cognition, to understand their effect on cognitive processing and the implications for learning/ education. She is currently PI on the EPSRC funded project 'Designing Tangibles for Learning: An empirical investigation'.

\section{Jennifer Sheridan}

Jennifer G. Sheridan is a Research Officer on the Designing Tangibles for Learning 
project, at the London Knowledge Lab. Jennifer holds a PhD in Computer Science from Lancaster University, and MS in $\mathrm{HCl}$ from Georgia Institute of Technology. Jennifer is cofounder of BigDog Interactive, a company of programmers and artists who create bespoke code for interactive installations and performance events. Her research interest is in Digital Live Art and she has published widely and exhibited her work in numerous public spaces. She founded the (re)Actor conference series.

\section{Taciana Pontual Falcão}

Taciana is a Research Officer on the Designing Tangibles for Learning project, at the London Knowledge Lab. Taciana holds a MS in Human-Computer Interaction from the Federal University of Pernambuco, Brazil. Before joining the Lab, Taciana was a researcher of the Cognitive Science and Educational Technology Group, Federal University of Pernambuco. She has worked at the Centre of Activities for Gifted Children (NAAH/S), and with Young and Adult Education in public schools in Recife. Taciana has a background in Computer Science, being specifically interested in the use and effects of Technology in Education, including accessibility issues and universal design.

\section{George Roussos}

George Roussos is a senior lecturer at Birkbeck College. He holds a B.Sc. In Mathematics from University of Athens, a M.Sc. in Numerical Analysis and Computing from University of Manchester and a Ph.D. from Imperial College, London. At Birkbeck he is currently leading the pervasive computing team, investigating the effects of social activity on system architectures, and exploring mechanisms to support navigation and findability. He is a member of the ACM, SIGMOBILE, the IEEE, the IEEE Communications and the IEEE Computer Society.

Abstract: External representations have been shown to play a key role in mediating cognition. Tangible environments offer the opportunity for novel representational formats and combinations, potentially increasing representational power for supporting learning. However, we currently know little about the specific learning benefits of tangible environments, and have no established framework within which to analyse the ways that external representations work in tangible environments to support learning. Taking external representation as the central focus, this paper proposes a framework for investigating the effect of tangible technologies on interaction and cognition. Key artefact-actionrepresentation relationships are identified, and classified to form a structure for investigating the differential cognitive effect of these features. An example scenario from our current research is presented to illustrate how the framework can be used as a method for investigating the effectiveness of differential designs for supporting science learning.

Keywords: Conceptual framework, tangible user interfaces, external representation, cognition, interaction, learning. 


\section{Introduction}

Tangibles, in the form of physical artefacts embedded with wireless, sensor and actuator technologies, offer the opportunity to flexibly combine digital information (e.g. sound, images, text) with artefacts (Zuckerman et al., 2005), the environment (Rogers et al, 2002; WAAG, 2005) or action (Raffle et al., 2006; Price and Rogers, 2003) to provide different information than is normally available in the immediate physical environment. Theories of learning and cognition offer a compelling rationale for using tangible and embodied interaction for supporting learning (e.g. O'Malley and Fraser, 2004), being compatible with socio-constructivist theoretical concepts including hands-on engagement; experiential learning (Bruner, 1979); construction of models (Papert, 1980; Resnick, 1998); collaborative activity and transformative communication (Pea, 1994). Successful application of tangible environments in various learning contexts has been demonstrated, e.g. narrative (Annany and Cassell, 2001), exploration and construction (Zuckerman et al., 2005; Raffle et al., 2006), models of phenomena (Moher, 2005), and pattern based interaction (Yonnemoto et al., 2006). The implications for learning are considerable, but little research offers any significant understanding of the specific learning benefits of tangible environments. Real learning gains from tangible interaction are being questioned (Marshall, 2007) and the need for rigorous empirical work is evident.

However, we currently lack an effective structure within which to establish such learning benefits. Marshall (2007) proposes six perspectives to guide research on tangibles for learning, but given the versatility of the 'tangible' space, a more detailed framework for structuring research within these perspectives is needed. Several frameworks for conceptualizing tangible environments have been described over the last decade, many focusing on taxonomies of systems, or as a basis for analysis (e.g. Ullmer and Ishii, 2001; Fishkin, 2004; Antle, 2007). None provide a framework for structuring systematic investigation of the cognitive effects of tangible environments. External representations are a central feature of tangible environments, and are well known to play an important role in cognition. The potential to exploit different forms of digital representations and flexibly link them with physical artefacts and action promises greater representational power. However, such flexibility of couplings brings with it an exponential number of factors for research. Extending our initial framework (Price, 2008), this paper presents a detailed framework for conceptualising tangible environments from an external representation perspective. Taking this as the core concept, the representation-tangible relationship is outlined, followed by 
the framework, which focuses on the relationship between different artefact-representation and action-representation combinations, and the role that they play in shaping cognition.

\section{Background}

\subsection{Tangible learning environments}

A number of tangible systems for learning in different contexts have been developed during the last decade. Studies of such systems primarily inform us about levels of engagement and enjoyment, and the technical achievements of mapping to learning activities that may be promoted through tangible interfaces. Concrete findings about learning gains are minimal, and the role of the representational relationships in supporting learning is rarely explicit. In this section, contributions from the field, with particular reference to the role of representation are explored.

Several tangible environments involve digitally embedding objects with e.g. LEDs or accelerometers. These have been used to communicate and show motion patterns (e.g. SystemBlocks, Resnick et al., 1998); generate visual representations of behaviour according to the way the objects are combined (e.g. Stackables, Resnick et al., 1998); record and transmit information about the object's own movement (e.g. Bitball, Resnick, 1998); and record and playback physical motion to facilitate children's learning about movement and locomotion (e.g. Topobo, Raffle, 2004). Collectively these digital manipulatives are claimed to be engaging, and enable 'natural' interaction through use of popular objects such as blocks and balls. Blocks have also been used for tangible programming, where users explore basic concepts of programming languages by arranging blocks with different functions (Wyeth and Purchase, 2002; Schweikardt and Gross, 2008). Again children were reported to show great interest, besides being able to use them without help. Although these systems demonstrate applications for relevant learning concepts, studies show little about their cognitive effectiveness and often focus on the artefact's capabilities for use in a variety of contexts (Raffle, 2004), or levels of engagement (Xie et al., 2008) rather than learning effectiveness.

Other tangible environments rely on direct mappings between physical action with objects and representations on computer screens, providing scaffolding and guidance according to children's actions (e.g. TICLE, Scarlatos et al., 1999; SmartStep and FloorMath, Scarlatos and Scarlatos, 2000). In contrast, other research suggests that different mappings between objects or action and representation can result in different kinds of cognitive 
activity, for example, introducing some level of ambiguity can provoke children's interest, curiosity and reflection (Price et al., 2003; Randell et al., 2004). A clearer understanding of the effect of different representational mappings in tangible environments is evident.

One application explicitly exploring different representational designs (Sensetable, Patten et al., 2001) showed that users preferred information displayed on the sensing surface rather than on a separate screen, precluding the need to divide their attention between the input (sensing surface) and the output (separate screen display). This is interesting in the context of our work, which seeks to go beyond user preference to understand the cognitive effect of such different designs.

\subsection{Tangible interface conceptual frameworks}

A number of frameworks and taxonomies for conceptualising tangible user interfaces are emerging. Primarily they provide a way of classifying tangible interfaces to describe, compare or analyse the different systems. However, none focus on defining the role of external representations in tangible environments or how they might be designed to support learning.

Ullmer and Ishii (2001), Koleva et al. (2003) and Fishkin (2004) all provide descriptive taxonomies, which formulate categories for configuration of different systems, but say little about the relative strengths and weaknesses of different designs in terms of interaction. Ullmer and Ishii (2001) provide a descriptive taxonomy for configuring and conceptualising the interactive space of tangible interfaces, but do not specify the different ways that physical-digital coupling can take place. Koleva et al. (2003) focus on computational coupling around the concept of degree of coherence i.e., how closely physical and digital objects, their links and properties map onto one another physically and conceptually. These features primarily take a systems-based perspective and do not include the physical design of the physical space nor the representational properties in any detail. Fishkin (2004) provides a taxonomy to locate, compare and integrate research in tangible interfaces defining them in terms of 'levels of tangibility' using two dimensions each with four levels: 'embodiment' specifies the relative distance between the physical and digital display; 'metaphor' specifies how close the effect of user action is to the real world effect of similar actions. These concepts are echoed in our framework, where the concept of tangibility is considered an important, but not sufficient, mediating factor, as it does not take into account the representational properties of the system. Furthermore, Fishkin's 
metaphor concept makes assumptions about interaction and cognition i.e., that direct mapping to the physical world using tools that we are currently familiar with is more powerful, than what might be termed indirect. As yet we know too little about the impact of other mappings on cognition or interaction to make any claims about their powerfulness. Nor do we know about the cognitive effect of the relationship between the level of embodiment and cognitive distance, to reliably inform design.

More recent theoretical approaches have placed a stronger focus on human interaction experience. Zuckerman et al. (2005) describe tangible interfaces in terms of implementation designs and related types of interaction. The taxonomy is useful for classifying types of interaction based on implementation options, but does not provide a means of analysing interactions and their effects in detail. Hornecker and Burr's (2006) approach encompasses design, interaction and bodily movement, highlighting the need to design physical tools and their interrelations as well as digital representations.

Few frameworks attempt to define tangible interfaces from the perspective of learning. Edge and Blackwell (2006) classify features of tangible environments in terms of their usability for programming languages, identifying design features through their physical properties of expression, but we know little about the impact of such design configurations on knowledge construction in various learning domains. Antle (2007) provides a descriptive analysis to inform the design of tangible systems for play-based applications, highlighting four areas of cognitive development, which tangible systems may support: embodied cognition; development of spatial cognition; development of symbolic reasoning; and development as a non-linear dynamic system, but advocates the need for more empirical evidence.

A detailed framework for empirical research is lacking. Indeed, the question of how to explore such environments and need for more principled approaches for supporting research and analysis of tangible environments are widely claimed (e.g. Dourish, 2001; Hornecker and Burr, 2006). External representations are particularly powerful cognitive tools, and their importance in conceptualizing the value of tangible environments for learning is central.

\subsection{External representation and cognition}

Theories of external cognition highlight the importance of external representations (Scaife 
and Rogers, 1996), and their function in supporting problem solving and learning through forms of computational offloading (e.g. Larkin and Simon, 1987; Bauer and Johnson-Laird, 1993). External representations that explicitly depict aspects of a problem are shown to ease problem solving, support the learner to make inferences, and free up cognitive activity to focus on relevant aspects of the task (Zhang and Norman, 1994). However, dynamic representations, being a transient media, show a more complex picture for cognition. Dynamic representations demand integration across representations (e.g. Stenning, 1998; Price, 2002), raising issues of increased memory load and subsequent impact on students' inferences (Price, 2002), multidimensionality (Price, 2002), integration of representations (e.g. Rogers and Scaife, 1998), and meaningful linking between physical interaction and abstract conception (Clements, 1999).

Much of this research has focused on visual representation, but the emergence of ubiquitous technologies has increased interest in the value of other representational media. Audio has been found to mediate understanding of large amounts of abstract data in complex systems (Childs, 2001; Garcia-Ruiz, 2001); and different modes of representation (descriptive, experimental, kinaesthetic, embodied) are thought to support students to handle a diversity of representations and identify conceptual links between them during scientific reasoning (Prain and Waldrip, 2006). Tangible environments also offer opportunities for capitalizing on physical representations in the form of artefacts (Marshall, 2007) conveying newer forms of information when combined with other forms of representation. Such features result in an inherently dynamic environment, where both physical and digital representations can change in form, space or time. Furthermore, explicit depiction of phenomena through external representations (physical and digital) may reduce cognitive computation and the active 'working out' that is instrumental for learning (Jones and Scaife, 2000).

Some research has begun to identify ways in which interaction and learning activity might be mediated by representation-device relationships. For example, Randell et al. (2004) showed that representations with more ambiguous mappings promoted higher levels of collaborative reflection through discussion than direct mappings; and Price et al. (2003) suggest the value of unexpected or unfamiliar events (between action or artefact and representation) for attracting attention and promoting reflection. The value of more idealized and less realistic representations has also been shown through empirical research. Despite the common belief that abstract concepts are more easily grasped 
through experience with concrete representations (being explicitly designed to support abstract reasoning), connection between physical objects and underlying abstract concepts is not always transparent to students (Goldstone and Son, 2005). Another aspect of concrete representations is the risk of them narrowing students' comprehension, limiting it to a specific context, and blinding them to the general nature of the abstract concept (Goldstone and Son, 2005). The key point here is how to combine the perceptual scaffolding provided by concrete materials with the necessary abstraction of more symbolic representations.

\subsection{Action, embodiment and representation}

In tangible environments external representations are not only coupled with objects, but also with the action that is placed upon them. Theories of embodied cognition argue that such actions are central to our understanding of the world, embodiment being "the property of our engagement with the world that allows us to make it meaningful" (Dourish, 2001:126). Embodied cognition describes how the mind, body and world work together and influence one another to create and manipulate meaning. The relationship between physical experience and cognition has been broadly demonstrated, for example, through Gibson's (1977) theory of affordance for action based on perception; the importance of sensori-motor experience in cognitive development (Piaget, 1972); enquiry-base discovery learning (Bruner, 1979) and the relationship between abstract concepts and bodily experience through metaphorical expression (Lakoff and Johnson, 1980).

Embodied cognition then, is a meaning-making process that occurs through embodied interaction. In tangible environments external representations are a central part of this interaction and meaning-making process. Although this concept of embodiment is becoming increasingly prominent in the underlying design of tangible environments, little focus is placed on the representation-action relationship. Antle (2007) suggests an embodied cognition perspective is particularly relevant for children where the interplay of action and cognition serves to improve both motor and cognitive processes. At a more concrete level Fishkin (2004) describes embodiment in terms of how much attention users need to pay to the device rather than what that device represents. He argues that as embodiment increases, the 'cognitive distance' between input and output increases, thus if it is important to maintain 'cognitive dissimilarity' between input and output 'objects' then the degree of embodiment should be decreased. This concept relates to the role of 'stepping in, stepping out' in learning (Ackerman, 1999) and notions of expressive versus exploratory interaction with tangibles (Marshall et al., 2003). 
To understand the value of tangible environments for learning it is essential to consider how action affects cognition, and gain a clearer understanding of the relationship between representation-action couplings, and the subsequent cognition e.g. in terms of inferences, conceptual understanding.

\section{The Framework}

Our framework builds on concepts from previous frameworks and seeks to address some of the issues raised by focusing the external representation-action-artefact relationships that occur in tangible environments as a way of conceptualising physical-digital links and analysing their effects from a learning perspective. Little research in tangible environments places external representation at its centre. A focus on representation offers the means to better understand the role of tangibles for learning, not only in terms of representation linked to artefacts but also in terms of the manipulative as representation, rather than as an object in itself. Physical manipulatives also require various actions to be performed so, not only is the object itself (as a representational entity) computationally coupled with digital information, but also the action or movement placed upon it. At the same time learners are required to make meaningful inferences and mappings between both artefacts and action. This framework provides a comprehensive focus on different physical-digital couplings that form a structure for exploring the different roles of external representations play in supporting learning in tangible environments. The location parameter refers to the distance in space between physical and digital components of the system; dynamics is related to the flow of information throughout the interaction, including links between action, intention, and feedback; and correspondence depicts the metaphors involved in the nature of representations of artefacts and actions upon them. The modality of representation impacts on different aspects of the whole interaction and is therefore considered in parallel to all other categories (see fig. 1).

\subsection{Location}

This parameter consists of three categories, which specify the location of representation in relation to the object, or action, in physical space. These location couplings are important for cognition in terms of making links between object, action and representation, and the effect of this both conceptually and on computational offloading. Key concepts include: attention demands required to make appropriate links e.g. whether distant coupling engenders more cognitive effort (hence improved reasoning) in making mappings between 
action and representation, or hinders meaningful cognitive interaction; ease of problem solving or making inferences through explicitness of representation e.g. whether location couplings affect levels of explicitness; the potential for representing multiple levels of abstraction and the related demand for integration across representations. Furthermore, the object-representation relationship has an impact on the kind of action that can take place. For example, co-located coupling action is essentially constrained to placing and moving objects on a surface and removing them from the surface. This contrasts with the broader kinds of movements facilitated through linking with discrete and embedded representations, and enables exploration of action possibilities for cognition (see 2.4 and 3.3).

\section{Discrete}

Input and output are located separately, i.e., a manipulated object triggers a digital representation on a separate screen display (fig.2). For example, Chromarium, a tangible environment to support children's exploration of colour mixing, used an adjacent digital display to show the effects of mixing colours on cubes embedded with RFID technology (Gabrielli et al., 2001).

\section{Co-located}

Input and output are contiguous, i.e., the digital effect is directly adjacent to the artefact or action (fig. 3). For example, Urp, a model urban planning environment displays effects of architectural structures, such as shadows or wind patterns, onto a surrounding horizontal table surface (Underkoffler and Ishii, 1999).

\section{Embedded}

A digital effect occurs within an object, e.g. the object lights up, moves, exerts force or 
changes shape or colour according to actions placed upon it (fig. 4). For example, Flow Blocks, embedded with sensors, send light signals through the blocks when connected together, to help children explore different causal structures (Zuckerman et al., 2006).

\subsection{Dynamics (information flow)}

This parameter consists of two categories that relate to the 'flow' of information through different networked links that occur between artefact and representation, and action and representation. The categories are based on characteristics of information flow that may have important consequences for learning, in particular the relationship between system causality and perceived causality (both in relation to action and conceptual phenomena); and the relationship between intentional information access and learning activity. Digital effects, or feedback, can be immediate or delayed, or may be dependent on multiple objects or interactions to be triggered. The resultant causal relationships can be quite complex, requiring better understanding of the impact of such flow of information on cognition. Embodied interaction also plays a role in dynamic information flow through intentional action and corresponding digital effects, and serendipitous triggering of information. Different information couplings have been shown to influence attention and reflection through 'unexpected' couplings, both in time and 'effect' (i.e representation). Inadvertent elicitation may attract attention (guiding learning) or engender unexpected discovery or discussion (Randell et al., 2004), but a clearer understanding of how learners integrate unexpected effects into their activity and the impact on attention and conceptual understanding is needed.

\section{Causality}

Defined according to system-based feedback associations allows investigation into the difference between system based causality and perceived causality, as well as the subsequent effect in conceptual causality (i.e. of the subject domain)

- Simple: Digital effect is immediate and constitutes a direct association between object/action and effect. For example, placing a cube on a surface makes a corresponding square appear on a separate screen.

- Complex: Occurs when representation and meaning are changing or developing over time through continued interaction with artefacts, and/or cumulative information

- recorded by the system from a series of events or learner interaction. Such feedback, dependent on multiple actions, often occurs with a time delay and may create an ambiguous association between object/action and effect. 
Intentionality

Embraces the user-control perspective of technology-based environments focusing on action-representation couplings to account for intentional and serendipitous information triggers.

- Intentional: Digital effects can occur contiguously with intentional action, generating an expected effect.

- Serendipitous: Digital effects inadvertently triggered through actions on objects according to pre-determined configurations often causing an unexpected effect.

\subsection{Correspondence}

This parameter refers to the metaphorical mappings between objects, representations and action and the learning concept. Metaphorical mappings can form the conceptual basis of ideas or phenomena. In tangible environments multiple metaphorical correspondences occur and interact with one another. Three correspondence categories focus on the physical object characteristics, representational mappings, and action mappings. Investigating how these mappings facilitate understanding of scientific concepts, or engender effective learning strategies (e.g. cognitive conflict, reflection, exploration) is essential for understanding how such correspondences can best support learning. For example, the effects of ambiguous versus direct mappings or the level of perceptual scaffolding between concrete materials and symbolic representations on computational offloading, ease of interpretation, and meaning making (see sec. 2.3).

\section{Physical correspondence}

This category refers to the degree to which the physical properties of the objects are closely mapped to the learning concepts. Although similar to structural correspondence (Edge and Blackwell, 2006), the emphasis here is on degree of correspondence to the metaphor of the learning domain, and how this constrains or influences inferences and conceptual understanding.

- Symbolic: defines objects that act as common signifiers, where the object may have little or no characteristics of the entity it represents e.g. a block could represent a book or abstract entities, like chromosomes or circuit components.

- Literal: defines objects whose physical properties are closely mapped to the metaphor of the domain it is representing, e.g. a rigid block representing 
chromosomes reveals none of the fragility that is inherent in the process of genetic changes, whereas loosely magnetically connected 'strips' could convey relevant underlying 'fragile' features.

\section{Representational correspondence}

This category encompasses design considerations of the representations themselves and their metaphorical mapping within the learning domain. Meaning mappings between physical and digital representations can be designed such that the representations themselves differ in levels of association (direct to ambiguous) between symbol and symbolised according to the concept being displayed. Ambiguous mappings are shown to engender different levels of reflection about meaning in context (Randell et al, 2004), but direct mappings may support computational offloading. This category is also concerned with understanding how to facilitate the grasp of abstract concepts for example, through the notion of perceptual scaffolding between the concrete materials and their related symbolic representations (Goldstone and Son, 2005).

\section{Action correspondence}

Gibson's (1977) theory of affordance describes the "action possibilities" that can be performed on an artefact in relation to an actor's physical capabilities. We broaden this view to include active sensorimotor exploration. Tangible environments offer opportunities for exploration, meaning-making and non-verbal expression through action. Such action is apparent not only through the movement itself but also through associated digital representations. A key question concerns the relationship between action, external representation and cognition, for example, understanding how different representations are interpreted in relation to particular kinds of action.

Actions can be impactive, requiring physical contact with an artefact, e.g. grasp and grip, or non-impactive, e.g. gesture. Here Sheridan et al.'s (2003) classification is expanded to include descriptions of movement, to enable investigation of the cognitive effect of different correspondences between physical action and related representation.

- Manipulation: is a type of action. A grasping manipulation might be "hold" or "press"; a gripping manipulation might be "squeeze" or "turn"; and, a gesture manipulation might be "moving hand left and right." Turning might be described as manipulating an artefact around its y-axis. Manipulations can be further sub-categorized into events. For example "rotation" is a subcategory of "turn" where rotating a cube with 
the fingers might be described as "to turn about the axis or centre in a continuous, fluid motion, exposing sides" (ibid).

- Movement: refers to the characteristics of the action being performed and include:

- Duration: whether a movement is discrete (e.g. pressing a key once) or continuous (rotating a dial)

- Flow: refers to speed of a movement (slow, fast) and whether it is jerky or smooth

- Regularity: describes the rhythm of movement which can be regular or irregular

- Directionality: describes movements up, down, left or right, and includes movement around an axis, e.g. rotating a cube requires consideration of twofold (vertex), three-fold (edges), and four-fold (faces) axis.

\subsection{Modality}

Although the visual mode is a predominant form of representation the potential for audio and tactile modes in tangible computing requires a broader understanding of their role for learning. A key issue is to understand the value of different dynamic representation modalities, and their effects when integrated with each other and with physical interaction. For example, verifying the conceptual links student make and to what degree different modalities convey newer forms of information (see 2.3).

\section{Visual}

Currently, most information presented by computers uses different forms of visual representations on screens, such as textual, graphic, tabular and pictorial (Prain and Waldrip, 2006). The visual modality allows large amounts of detailed information to be displayed. However, information can be missed if the user is not looking to the right place at the right time or due to screen overload (Brewster, 2002).

\section{Tactile}

Our skin possesses a high sensitivity that conveys a rich understanding of objects we touch (Hoggan et al., 2007), recognizing texture and detecting slip, flexibility and direction of edges. With the development of touch as an interaction technique, skin can be used as a medium to communicate information (Brewster and Brown, 2004). Touch is kinaesthetic (information arising from forces and positions sensed by the muscles and joints) and cutaneous (sensations of vibration, temperature, pain and indentation). Also, 
information can be encoded according to different vibro-tactile parameters such as rhythm, roughness, intensity, frequency and spatial location (Brown et al., 2006).

\section{Audio}

While our visual sense provides detailed information about a relatively small area of focus, our auditory system captures general information from all around, directing our attention to things outside our vision (Brewster, 2002). Verbal sound is already common for narrative and instructions in multimedia applications. Non-speech sound is used as alarms and warnings (calling attention to some event) or in multimedia applications as music and sound effects (creating an ambiance). Using sound to convey information may reduce screen overload, grab attention more easily and represent some objects or actions more naturally. However, presenting absolute data with sound is difficult (often dependent on subjective interpretation); information is transient and must be remembered or replayed by user; and auditory feedback can become annoying with repetition (Brewster, 2002).

This framework essentially illustrates the different representational possibilities associated with artefacts and actions in tangible systems. It aims to provide a structure within which research can be positioned rather than provide a prescriptive comparative system for research, which demands consistency of metaphor across the tangible interfaces. The different artefact-action-representation relations in tangible interfaces inherently contain and lend themselves to different metaphorical associations.

\section{Working with the framework}

The framework presented in this paper forms the basis for the EPSRC funded Designing Tangibles for Learning project, which aims to systematically investigate and understand how different ways of linking together objects, environments and information affect the way that learners interact with and understand scientific ideas. A purpose built reactable environment (Jordà, 2003), together with digitally embedded objects and a separate screen display will be used to investigate aspects of the framework with students learning of scientific phenomena.

Applications are based on children's common misconceptions of scientific concepts involving physical phenomena whose underlying theory is not apparent in the everyday world. For purposes of illustration we present designs to support reasoning about colour concepts in relation to reflection, absorption and transmission of light waves. In this application a set of different-coloured objects and an object to represent a light source 
(e.g. a torch) will be used. Below we give a simple example illustrating our study design for investigating 'location' (section 3.1), where the same concepts are conveyed through different representations to enable analysis of the cognitive and interactive effects of each.

- Discrete: users manipulate the objects on the sensing surface and see the effects of their actions on a separate screen. For example, when the torch is pointed to a red object on the surface, representations of the torch beam and the reflected red light rays bouncing off the object will appear on a vertical screen. Absorbed light frequencies could be shown by illuminating the on-screen representation of the object with the corresponding colours or showing a rainbow-like representation around the object on the screen (fig. 5).

- Co-located: users manipulate the objects on the sensing surface, and corresponding effects are shown through a projector placed under the table as well. In this case, the user can see the reflected light rays on the table itself, i.e. in a contiguous space and the absorbed light as a rainbow-like representation around the concrete object (fig. 6).

- Embedded: users freely manipulate a transparent object and the torch. The object itself is set to simulate the behaviour of a coloured object. When directing light the object reacts by emitting light back to the user to represent the reflected rays. Furthermore, using a hole on the top of the object, users can look inside the object and see an area illuminated by the colours it is absorbing (fig. 7).

Studies will investigate the relationship between different locations and aspects like:

- attention demands (whether having a separate screen for feedback requires a specific shift of attention and how this affects reflection and comprehension);

- ease of making inferences (e.g. whether location couplings affect explicitness of representation);

- levels of abstraction (with combined locations representing local and global effects of phenomena) e.g. effect on ease of integration across representations and understanding of causality

- action (meaning-making through mappings between action and representation).

Further aspects of the framework can then be built in for future studies. For example, in terms of physical correspondence general-shaped objects e.g. cubes (symbolic) and real world objects e.g. a wooden bowl, a yellow tennis ball (literal) can be used. As real-world 
objects are made of different materials, this will form a key aspect when analysing children's reasoning about light behaviour. Representational correspondence mappings will vary according to modality. For example, objects could react by emitting different sounds according to the concepts in question, introducing ambiguous representational correspondence, while we expect that visual representations will have more direct mappings to the metaphor of the underlying theories of light behaviour. Our study design will begin with the example above, focusing on location, followed by variation of the other parameters to analyse the consequences of each one.

\section{Conclusion}

Recent technology development makes the combining of digital information with physical artefacts, environment and action more feasible, providing new possibilities for conveying and representing information. This shows particular promise for education, enabling hands-on activity to be enhanced through various forms of digital augmentation and offers the potential for learners to act and think at different levels of abstraction through the combinations of concrete manipulatives and more abstract or more formal representations of concepts they are learning. However, little research offers significant insight into the cognitive effects of tangible environments or provides the means for structuring such investigation. This paper proposed a framework for conceptualising tangible environments from an external representation perspective, and for structuring related research. External representations are central to tangible environments, which have potential to exploit different forms of digital representations, and link them with physical artefacts and action. The flexibility of such couplings gives rise to a number of different representational relationships that form the basis of the framework. These representational features are identified in terms of: location (location of representation in relation to the object, or action, in physical space); dynamics (different information associations or the networked links and 'flow' of information that occur between artefact and representation, and action and representation); correspondence (concerning metaphorical mappings between objects, representations and action and the learning concept); and modality (the different representation modalities that can be used in conjunction with physical interaction). As part of the current research, the value of this framework will be assessed and iteratively refined.

\section{Acknowledgements}

This research is supported by the EPSRC: Grant number EP/F018436 


\section{Bibliography}

Ackerman, E. (1999) Enactive Representations in Learning: Pretense, Models, and Machines. In Bliss, J., Light, P. and Saljo, R. eds. Learning Sites: Social and technological Contexts for learning, Elsevier, 144-154.

Annany, M. and Cassell, J. (2001) Telling Tales: A new toy for encouraging written literacy through oral storytelling. Presentation at Society for Research in Child Development, Minneapolis.

Antle, A. Designing Tangibles for Children: What Designers Need to Know (2007a). Proceedings of $\mathrm{CHI}$ 2007, Work in Progress, ACM Press.

Antle, A. The CTI framework: informing the design of tangible systems for children. (2007b) Proceedings of the 1st International Conference on Tangible and Embedded Interaction, ACM Press 195-202.

Bauer, M. and Johnson-Laird, P. (1993) How diagrams can improve reasoning?

Psychological Science 4, (6), 372-378.

Brewster, S.A. Chapter 12: Non-speech auditory output. (2002) In Jacko, J. and Sears, A. (Eds.) The Human Computer Interaction Handbook (Lawrence Erlbaum Associates, USA) pp 220-239. ISBN 0-8058-4468-6.

Brewster, S.A. and Brown, L.M. (2004) Tactons: Structured Tactile Messages for Non Visual Information Display. Proceedings of Australasian User Interface Conference (Dunedin, New Zealand), Australian Computer Society, pp15-23.

Brown, L.M., Brewster, S.A. and Purchase, H.C. (2006) Multidimensional Tactons for NonVisual Information Display in Mobile Devices. Proceedings of MobileHCl (Espoo, Finland), ACM Press, pp231-238).

Bruner, J.S. (1979). On Knowing. Cambridge, MA.: Belknap Press of Harvard University Press System.

Childs, E. (2001) The Sonification of Numerical Fluid Flow Simulations. Proceedings of the 2001 International Conference on Auditory Display, Finland 2001.

Clements, D. (1999) 'Concrete' Manipulatives, Concrete Ideas. Contemporary Issues in Early Childhood, 1, 1, 45-60.

Dourish, P. (2001) Where The Action Is: The Foundations of Embodied Interaction. MIT Press.

Edge, D. and Blackwell, A. (2006) Correlates of the cognitive dimensions for tangible user interfaces. Journal of Visual Languages and Computing, 17 (4), 366-394.

Fishkin, K. P. (2004). A taxonomy for and analysis of tangible interfaces. Personal and Ubiquitous Computing, 8 (5), 347-358

Gabrielli, S., Harris, E., Rogers, Y., Scaife, M. \& Smith, H. (2001) How many ways can you mix colour? Young children's explorations of mixed reality environments. CIRCUS 2001 Garcia-Ruiz, M.A. (2001). Using non-speech sounds to convey molecular properties in a virtual environment International Conference of New Technologies in Science Education (CINTEC), Aveiro, Portugal, 4-6 July 2001

Gibson, J. J. The Theory of Affordances. (1977) In Perceiving, Acting, and Knowing, Eds. Robert Shaw and John Bransford.

Goldstone, R. L.; Son, J. Y. (2005) The Transfer of Scientific Principles Using Concrete and Idealized Simulations. The Journal of the Learning Sciences, 14 (1), 69-110.

Hoggan, E., Brewster, S.A. and Anwar, S. (2007) Mobile Multi-Actuator Tactile Displays. In Proceedings of 2nd International Workshop Haptic and Audio Interaction Design (Seoul, Korea). Springer LNCS, pp 22-33.

Hornecker E, and Buur, J. (2006) Getting a Grip on Tangible Interaction: A Framework on Physical Space and Social Interaction. Proceedings of $\mathrm{CHI}$ 2006. Montreal. ACM Press, 437-446.

Jones, S. (aka Price) and Scaife, M. (2000) Animated Diagrams: An investigation into the cognitive effects of using animation to illustrate dynamic processes. In M. Anderson \& P. 
Cheng (eds) Theory \& Applications of Diagrams. Lecture Notes in Artificial Intelligence, no. 1889, Berlin, Springer-Verlag. pp. 231-244.

Jordà, S. (2003) Sonigraphical Instruments: From FMOL to the reacTable. Proceedings of the $3^{\text {rd }}$ Conference on New Interfaces for Musical Expression (NIME 03), Montreal

(Canada)

Koleva, B, Benford, S, Hui Ng, K. and Rodden, T. (2003) A Framework for Tangible User

Interfaces. Physical Interaction Workshop on Real World User Interfaces. Mobile $\mathrm{HCl}$,

Udine, Italy.

Lakoff, G. and Johnson, M. (1980). Metaphors we live by. Chicago: University of Chicago

Press.

Larkin, J. and Simon, H. (1987) Why a diagram is (sometimes) worth ten thousand words Cognitive Science 11, 69-100.

Marshall, P., Price, S., and Rogers, Y. (2003). Conceptualising tangibles to support

learning. Proceedings of Interaction Design and Children, Preston, England, July 1-3, pp

101-110.

Marshall, P. (2007) Do tangible interfaces enhance learning? Proceedings of First International Conference on Tangible and Embedded Interaction, USA.

Moher, T., Hussain, S., Halter, T., and Kilb, D. (2005). RoomQuake: Embedding dynamic phenomena within the physical space of an elementary school classroom. Conference on Human Factors in Computing Systems. 1655-1668, ACM Press.

O'Malley, C. and Fraser, D. S. (2004). Literature review in learning with tangible technologies. Technical Report 12, NESTA Futurelab.

Papert, S. (1980) Mindstorms: Children, computers and powerful ideas. NY, Basic Books.

Pea, R. D. (1994). Seeing what we build together: Distributed multimedia learning

environments for transformative communications. Journal of the Learning Sciences, 3 (3), 285-299

Piaget, J. (1972). The psychology of the child. New York: Basic Books.

Prain, V., and Waldrip, B. (2006) An Exploratory Study of Teachers' and Students' Use of Multimodal Representations of Concepts in Primary Science. International Journal of

Science Education, vol 28, no 15, 1843-1866

Price, S. (2002) Animated Diagrams: How effective are explicit dynamics for learners? In P. Bell, R. Stevens, and T. Satwitz (eds), Keeping Learning Complex: The Proceedings of the Fifth International Conference of the Learning Sciences (ICLS) 344-351. Mahwah, NJ:

Erlbaum.

Price, S. (2008) A Representation Approach to Conceptualising Tangible Learning

Environments. Proceedings of the Second International Conference on Tangible and

Embedded Interaction, TEl'08. Bonn, Germany

Price, S. and Rogers, Y. (2003) Let's get physical: the learning benefits of interacting in digitally augmented physical spaces. In (eds) J. Underwood and J. Gardner, Computers and Education: Special issue: 21st Century Learning: 43, 137-151

Price, S., Rogers, Y., Scaife, M., Stanton, D. and Neale, H. (2003) Using 'tangibles' to promote novel forms of playful learning. Interacting with Computers, 15/2, May 2003, 169185.

Raffle, H. (2004) Topobo: A 3-D Constructive Assembly System with Kinetic Memory. Master's thesis, School of Architecture and Planning, M.I.T., USA.

Raffle, H., Parkes, A., Ishii, H. and Lifton, J. (2006) Beyond record and play. Backpacks:

Tangible Modulators for Kinetic Behavior. CHI 2006 April, Montreal, Canada.

Randell, C. Price, S. Rogers, Y. Harris. E. and Fitzpatrick, G (2004) The Ambient Horn:

Designing a novel audio-based learning experience, Personal and Ubiquitous Computing. 8, 3, 144- 161.

Resnick, M. (1998). Technologies for lifelong kindergarten. Educational Technology Research and Development, 46 (41). 
Resnick, M., Maryin, F., Berg, R., Boovoy, R., Colella, V., Kramer, K., et al. (1998). Digital manipulatives: new toys to think with. Proceedings of the SIGCHI Conference on Human Factors in Computing Systems (CHI'98), pp 281-287, Los Angeles, USA.

Rogers, Y. and Scaife, M.(1998) How can interactive multimedia facilitate learning? In Lee, J.(ed.) Intelligence and Multimodality in Multimedia Interfaces: Research and Applications. AAAl.

Rogers, Y., Scaife, M., Harris, E., Phelps, T., Price, S., Smith, H., et al. (2002). Things aren't what they seem to be: Innovation through technology inspiration. Proceedings of DIS'2002 Designing Interactive Systems (pp. 373-379). New York: ACM Press Scaife, M and Rogers, Y (1996) External Cognition: how do graphical representations work? International Journal of Human-Computer Studies 45, 185-213.

Scarlatos, L. L., Dushkina, Y., and Landy, S. (1999). TICLE: A tangible interface for collaborative learning environments. Extended Abstracts of the SIGCHI Conference on Human Factors in Computing Systems (CHI'99), pages 260-261, Pittsburgh, USA. Scarlatos, T. and Scarlatos, L. (2000). Tangible Math Applications. Available at: $<$ http://www.cs.sunysb.edu/ tony/TSresearch.htm>. Accessed on: 28 March 2007 Sheridan, J.G., Short B.W., Kortuem, G., VanLaerhoven K. and Villar, N. (2003) Exploring Cube Affordance: Towards A Classification Of Non-Verbal Dynamics Of Physical Interfaces For Wearable Computing. Proceedings of the SIGCHI Conference on Human Factors in Computing Systems (CHI'04), Vienna, Austria. ACMIEE Eurowearable 2003, pp. 113-118, Schweikardt, E. and Gross, M. (2008). The robot is the program: Interacting with roBlocks. Proceedings of the Second International Conference on Tangible and Embedded Interaction, TEl'08. Bonn, Germany.

Stenning, K. (1998) Distinguishing Semantic from Processing Explanations of Usability of Representations: Applying Expressiveness Analysis to Animation. In (eds.) J. Lee, Intelligence and Multimodality in Multimedia Interfaces: Research and Applications, AAAI Press.

Ullmer, B. and Ishii, H. (2001) Emerging frameworks for tangible user interfaces. Carroll, J. ed. Human Computer Interaction in the New Millenium, Allison-Wesley, 2001, 579-601. Underkoffler, J. \& Ishii, H. (1999) Urp: A Luminous-Tangible Workbench for Urban Planning and Design Proceedings of CHI, May 15-19 WAAG Society, (2005) last retrieved April 2008 http://freq1550.waag.org/

Wyeth, P. and Purchase, H. C. (2002). Tangible programming elements for young children. Proceedings of the SIGCHI2nd International Conference on Human Factors in Computing Systems (CHI'02), pages 774-775, Minneapolis, USA.

Xie, L., Antle, A., Motamedi, N. (2008) Are Tangibles More Fun? Comparing Children's Enjoyment and Engagement Using Physical, Graphical and Tangible User Interfaces. Proceedings of the Second International Conference on Tangible and Embedded Interaction, TEl'08. Bonn, Germany.

Yonemoto, S., Yotsumoto, T. and Taniguchi, R. (2006) A Tangible Interface for Hands-on Learning, Tenth International Conference on Information Visualisation iv, 535-538.

Zhang, J. and Norman, D. (1994) Representations in distributed cognitive tasks. Cognitive Science 18, 87-122.

Zuckerman, O., Saeed, A., and Resnick, M. (2005). Extending tangible interfaces for Education: digital Montessori-inspired manipulatives. Proceedings of the SIGCHI Conference on Human Factors in Computing Systems (CHI'05), pages 859-868, Portland, USA.

Zuckerman, O. Grotzer, T. \& Leahy, K. (2006) Flow blocks as a conceptual bridge between understanding the structure and behavior of a complex causal system. Proceedings of the $7^{\text {th }}$ International Conference of the Learning Sciences Indiana, 880-886. 


\section{Figures}

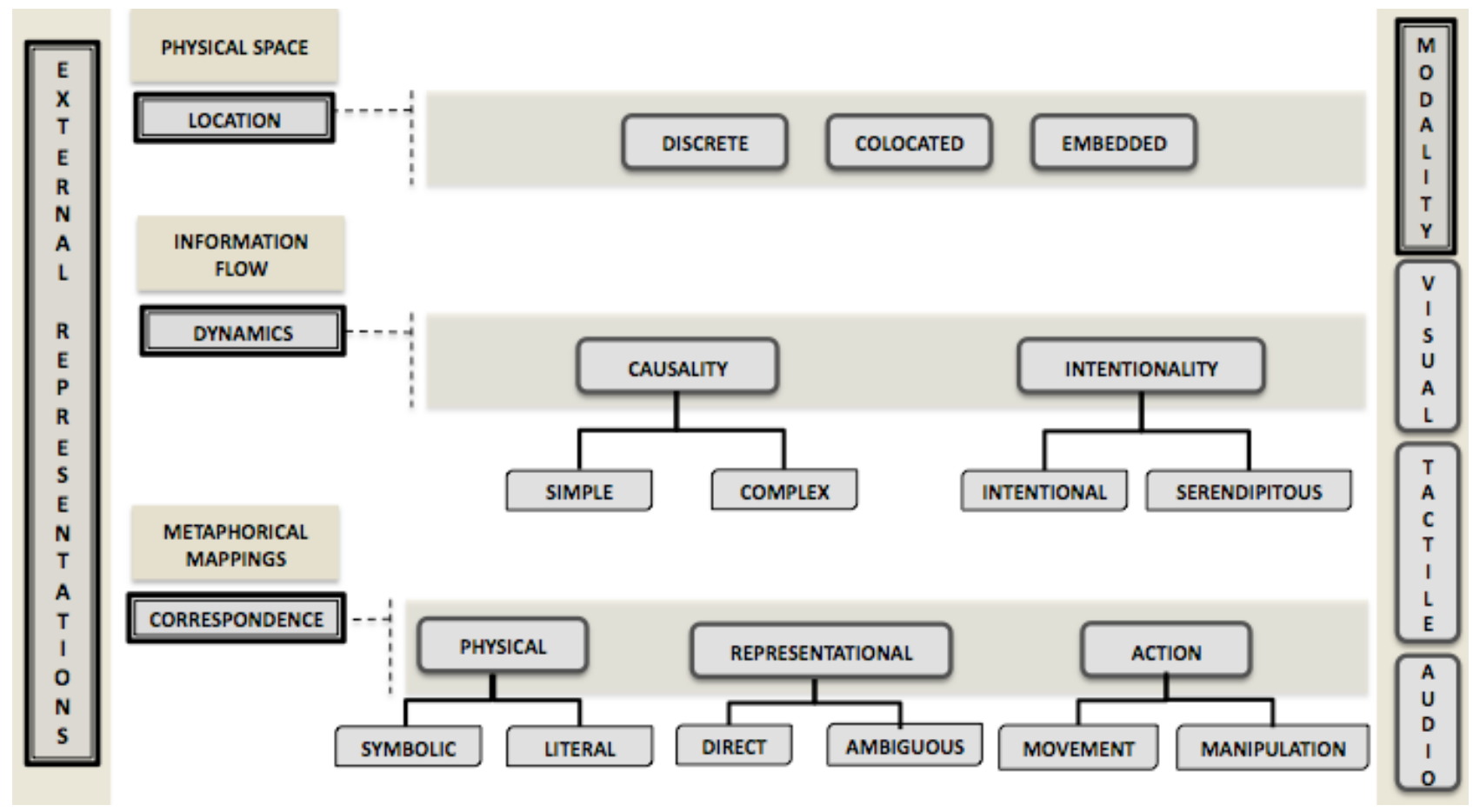

Figure 1 - Schematic overview of the framework

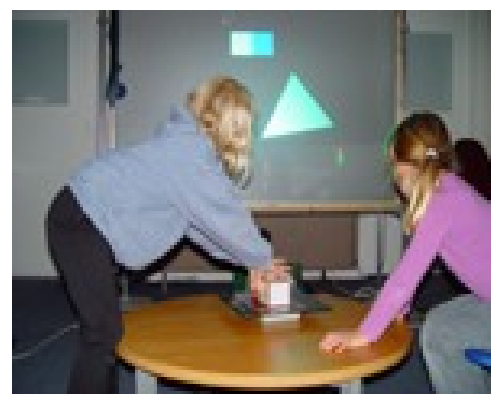

Fig. 2 - Chromarium (Gabrielli at al., 2001)

Fig. 3 - Urp (Underkoffler and Ishii, 1999)

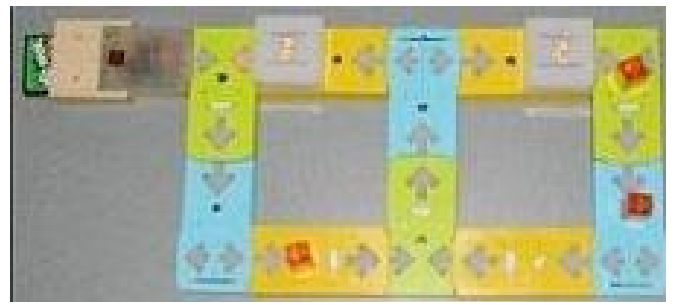

Fig. 4 - Flowblocks (Zuckerman et al., 2006) 


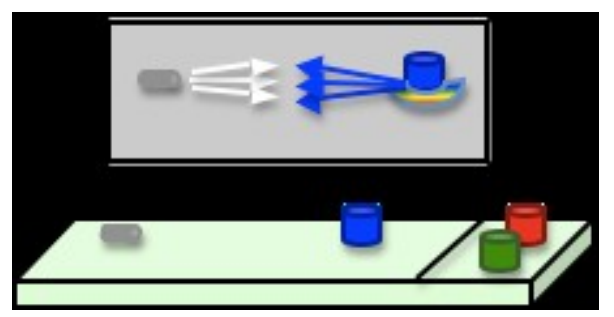

Fig 5 - Schematic representation of the discrete approach

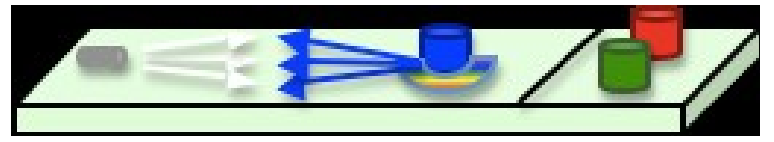

Fig. 6 - Schematic representation of co-located approach

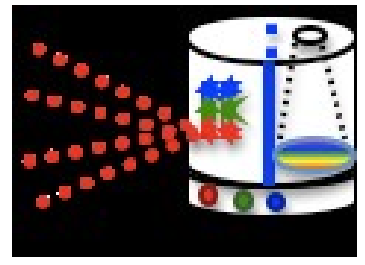

Fig. 7 - Schematic representation of embedded approach 